\title{
Numerical methods for random and stochastic partial differential equations
}

\author{
Alexey Chernov ${ }^{1}$ - Arnaud Debussche ${ }^{2}$. \\ Fabio Nobile ${ }^{3}$
}

Mathematical models involving partial differential equations (PDE) arise in numerous applications ranging from Natural Sciences and Engineering to Economics. Random and stochastic PDE models become very powerful (and sometimes unavoidable) extensions of deterministic models when a coefficient or forcing term cannot be adequately described by a single realization, e.g. due to their natural spatial or temporal variability, or the lack of information. This advantage comes at the price that stochastic models are usually significantly more difficult to solve and their numerical simulations result often in prohibitive computational costs. Feasible and reliable computer simulations require mathematically rigorous systematic studies of stability, convergence and efficiency of numerical algorithms and approximation methods.

This special issue collects contributions to the development and analysis of numerical approximation methods for both random and stochastic PDEs. The first three papers of this special issue are based on scientific results, and subsequent developments, reported at the workshop Numerical Methods for Uncertainty Quantification held at the Hausdorff Center for Mathematics (HCM), University of Bonn, Germany, in May 2013 and aimed at showcasing different aspects related to Uncertainty Quan-

$\bowtie \quad$ Alexey Chernov

alexey.chernov@uni-oldenburg.de

Arnaud Debussche

arnaud.debussche@ens-rennes.fr

Fabio Nobile

fabio.nobile@epfl.ch

1 Institute for Mathematics, Carl von Ossietzky University, 26111 Oldenburg, Germany

2 IRMAR et ENS Rennes, Campus de Ker Lann, Av. R. Schuman, 35170 Bruz, France

3 CSQI-MATHICSE, Ecole Polytechnique Fédérale de Lausanne, 1015 Lausanne, Switzerland 
tification in differential models and the most recent and important progresses in the field both at the theoretical and computational level.

One of the most promising techniques to compute statistics of the solution of a random or stochastic PDE is offered by multilevel Monte Carlo method. Three of the contributions in this special issue are devoted to improvements and extensions of this technique for random PDEs. In particular

- The work by C. Bierig and A. Chernov extends the general framework of the multilevel Monte Carlo method to estimate arbitrary order central statistical moments of the solution of a random PDE, showing that under certain assumptions the total cost of an MLMC central moment estimator is asymptotically the same as the cost of the multilevel sample mean estimator. The proposed technique is applied to a class of obstacle problems with rough random obstacle profiles.

- The work by I. Graham, R. Scheichl and E. Ullmann focuses on single phase flow problems in random porous media described by correlated lognormal permeability distributions. Such model is of interest, for instance, in radioactive waste disposal studies. A convergence and complexity analysis of the multilevel Monte Carlo method combined with a mass-conservative lowest order Raviart-Thomas mixed finite element approximation of the underlying PDE is presented.

- The work by A-L. Haji Ali, F. Nobile, E. von Schwerin and R. Tempone investigates optimal discretization hierarchies in the multilevel Monte Carlo method. The authors show that optimized hierarchies with geometric sequences of mesh sizes, are nearly as good and have the same asymptotic computational complexity as nongeometric optimal hierarchies. They also discuss the optimal tolerance splitting between the bias and the statistical error contributions and its asymptotic behavior. The optimized MLMC method is tested on a three-dimensional elliptic PDE with random but smooth coefficient and forcing term, as well as on a one-dimensional Itô stochastic differential equation.

The other two papers focus, instead, on stochastic parabolic or hyperbolic PDEs driven by white noise in time and establish convergence results of space-time discretizations. In particular

- The work by A. Anderson, R. Kruse and S. Larsson proposes a new way of proving weak convergence with essentially optimal rate for numerical approximations in space and time, combining standard Galerkin finite elements and backward Euler timestepping, of semilinear parabolic stochastic evolution equations driven by Gaussian additive noise, white in time and possibly colored in space. The new method of proof uses refined Sobolev-Malliavin spaces and does not rely on the use of the Kolmogorov equation or the Itô formula and is therefore non-Markovian in nature.

- The work by C. Bauzet, J. Charrier, and T. Gallouët considers a monotone finite volume discretization of multi-dimensional nonlinear scalar conservation laws forced by a multiplicative white noise in time and establishes convergence of the numerical scheme towards the unique stochastic entropy solution of the equation under appropriate stability condition on the time step. 